\title{
Global music approach to persons with dementia: evidence and practice
}

\author{
This article was published in the following Dove Press journal: \\ Clinical Interventions in Aging \\ 6 October 2014 \\ Number of times this article has been viewed
}

\author{
Alfredo Raglio ${ }^{1,2}$ \\ Stefania Filippi ${ }^{2}$ \\ Daniele Bellandi ${ }^{3}$ \\ Marco Stramba-Badiale ${ }^{4}$ \\ 'Department of Public Health, \\ Experimental and Forensic Medicine, \\ University of Pavia, Pavia, Italy; \\ ${ }^{2}$ APSP "Margherita Grazioli", \\ Povo, Trento, Italy; ${ }^{3}$ Geriatric \\ Department, Sospiro Foundation, \\ Sospiro, Cremona, Italy; ${ }^{4}$ Department \\ of Geriatrics and Cardiovascular \\ Medicine, IRCCS Istituto Auxologico \\ Italiano, Milan, Italy
}

\begin{abstract}
Music is an important resource for achieving psychological, cognitive, and social goals in the field of dementia. This paper describes the different types of evidence-based music interventions that can be found in literature and proposes a structured intervention model (global music approach to persons with dementia, GMA-D). The literature concerning music and dementia was considered and analyzed. The reported studies included more recent studies and/or studies with relevant scientific characteristics. From this background, a global music approach was proposed using music and sound-music elements according to the needs, clinical characteristics, and therapeutic-rehabilitation goals that emerge in the care of persons with dementia. From the literature analysis the following evidence-based interventions emerged: active music therapy (psychological and rehabilitative approaches), active music therapy with family caregivers and persons with dementia, music-based interventions, caregivers singing, individualized listening to music, and background music. Characteristics of each type of intervention are described and discussed. Standardizing the operational methods and evaluation of the single activities and a joint practice can contribute to achieve the validation of the application model. The proposed model can be considered a low-cost nonpharmacological intervention and a therapeutic-rehabilitation method for the reduction of behavioral disturbances, for stimulation of cognitive functions, and for increasing the overall quality of life of persons with dementia.
\end{abstract}

Keywords: music, music therapy, dementia, global music approach in dementia, evidencebased practice

\section{Background}

Music has been widely used in the context of dementia for many years now. ${ }^{1-5}$ Clinical experiences, different studies, and research have shown that the use of sound elements in dementia can be observed in a very wide and heterogeneous range of applications. Many different approaches and techniques are being used. This heterogeneity reflects the numerous possibilities of interventions with music in the treatment of persons with dementia (PWD). Music is an important resource for achieving psychological, cognitive, and social goals. However, current literature does not provide any systematic and precise overviews of these interventions, and sometimes omits to accurately describe the musical activities and the way in which they are employed, the clinical characteristics of the PWD (eg, type of dementia and level of severity), and the methods by which outcomes are evaluated. ${ }^{3,6}$

This paper describes the different types of evidence-based interventions that can be found in the literature and proposes a structured intervention model (global music approach for persons with dementia [GMA-D]). In this model, different music therapy approaches coexist, are integrated, and are clearly defined on the basis of content and
Correspondence: Alfredo Raglio

Department of Public Health,

Experimental and Forensic Medicine,

University of Pavia, Via Boezio 24, Pavia

27100 , Italy

Tel +390382593797

Email alfredo.raglio@unipv.it submit your manuscript | www.dovepress.com

Dovepress

http://dx.doi.org//0.2147/CIA.S71388

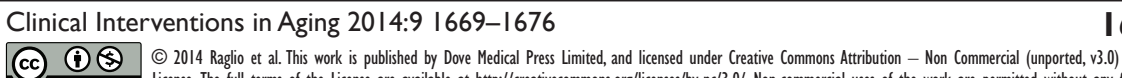
License. The full terms of the License are available at http://creativecommons.org/licenses/by-nc/3.0/. Non-commercial uses of the work are permitted without any further permission from Dove Medical Press Limited, provided the work is properly attributed. Permissions beyond the scope of the License are administered by Dove Medical Press Limited. Information on
how to request permission may be found at: http://www.dovepress.com/permissions.php 
application criteria. Current literature does not report any experience in which music was used by combining several techniques into one single approach for dementia; instead, it limits itself to describing the application of single techniques only.

In view of the heterogeneity of the approaches, which may vary according to place and culture, agreement on the definitions of the musical interventions appears to be of primary importance in order to achieve adequate implementation and repeatability ${ }^{7}$ of the experience.

This paper is organized in different sections that address the following issues: 1) identification of the theoretical and conceptual basis of the use of music in dementia, 2) definition of the music interventions available in literature, 3) identification in literature of evidence-based approaches 8 in favor of these interventions, 4) presentation of GMA-D, and 5) critical points and feasibility of the implementation of the proposed model.

\section{Theoretical and conceptual basis}

The impact of music has solid neuroscientific and psychological bases. Studies available in literature show how sound exerts a significant action on the brain ${ }^{9-13}$ by involving large cortical and subcortical areas and, particularly, the limbic and paralimbic areas that are responsible for the perception and elaboration of emotions. ${ }^{14}$ Music also stimulates motor and cognitive activation $^{15,16}$ at several levels, thus becoming an efficient instrument for rehabilitation. A recent review by Chanda and Levitin ${ }^{17}$ documents the effects of music at a neurochemical level by the involvement of some important circuits, such as those of recompensation and pleasure, of stress and arousal, of the immune system, and of social relations.

Sound and music have also been shown to achieve clear effects on vital parameters like blood pressure, heart rate, respiratory rate, and heart rate variability. ${ }^{18-21}$

From a psychological point of view, music facilitates communicative and relational processes and emotional expression. The literature on music therapy has defined the importance of this music-sound element, putting it at the base of the intersubjective development of each individual. ${ }^{22-24}$

In the field of dementia, several articles document the efficacy of music in the treatment of behavioral and psychological symptoms of dementia, ${ }^{25-27}$ in enhancing communications and relational processes, ${ }^{26}$ but also in increasing some cognitive functions such as memory, executive functions, speech, and attention. ${ }^{28-30}$

Other studies describe how cognitive functions that are related to music remain stable for a longer period of time compared with others, ${ }^{31-33}$ and this supports the potential impact of sound elements even in the presence of significant cognitive decline.

\section{Music interventions and the evidence}

For this paper we considered and analyzed the literature, starting from reviews and systematic reviews (English language, published in peer-reviewed journals, 2000-2014 period), concerning music and dementia. PubMed, PsycInfo, and Embase databases were searched using the following key words: "music", "music therapy", "music listening", "singing", "dementia", and "Alzheimer". 1-5,27,34-43

The following evidence-based fields of intervention emerged from the analysis of the literature:

- Active music therapy (psychological and rehabilitative approaches [AMT-PA] and [AMT-RA])

- Active music therapy with family caregivers and PWD (AMT-FC)

- Music-based interventions (MBI)

- Caregivers singing (CS)

- Individualized listening to music (ILM)

- Background music (BM).

From the clinical practice point of view, each of these interventions is based on a clear definition: 1) practical prerequisites, 2) used methods, and 3 ) possible goals according to specific clinical needs. Referring to "practice concepts", most attention was paid to the subject of the interventions, rather than to their results.

Music therapy interventions in the scientific culture of the discipline are characterized by a relational component, which is considered essential, and by the presence of a qualified music therapist. In these approaches, the treatment is therefore mediated by a therapist who uses applicable models based on psychological (AMT-PA and AMT-FC) and/or rehabilitative (AMT-RA) approaches. The psychological approach is based on the active techniques (which are based on direct interactions with the PWD using chiefly musical improvisation) that aim at reducing psychological symptoms or complications and at increasing relational and communication skills. Receptive techniques in music therapy involve verbal and elaborative competences that a PWD might, unfortunately, have lost. Rehabilitative music therapy models are mainly based on the use of active techniques, such as music exercises that constitute an effective motor, cognitive, and sensory rehabilitation tool. Music therapy also presents some other basic conditions that characterize a therapeutic intervention: a reference model that defines the theoretical and practical details that support the intervention, 
the presence of a therapeutic setting, the possibility to define targets and therapeutic strategies of intervention with adequate awareness, and the possibility to verify the therapeutic results achieved. In the clinical practice, music therapy approaches, but also other music interventions with different and specific characteristics, can be found, such as generic MBI, CS, ILM, and BM.

Reported studies regarding each intervention of GMA-D refer to more recent studies and/or studies with relevant scientific characteristics (where present) (mostly randomized controlled trials [RCTs] or clinical controlled trials $[\mathrm{CCTs}])$. The quoted studies can be considered significant examples of the clinical practice of the described approaches.

AMT can be defined as an approach that involves making music with the PWD (individually or in small groups) based on theoretical and practical assumptions of both a psychological and a neurocognitive nature. From the psychological point of view, AMT refers to relational therapeutic methods that may facilitate the processes of communication, expression, and modulation/control of the emotional-affective aspects of dementia. With regards to AMT with a neurocognitive basis, the approach is oriented toward aspects that are related to cognitive rehabilitation and tries to support several functions such as memory, speech, and executive functions. During relational interventions, sound and music are mainly employed referring to improvisational techniques (generally in the nonverbal context), but also with the use of structured material (eg, well-known songs or melodies); in the rehabilitation approach, sound and music are used in specific musical tasks. AMT requires the presence of a trained music therapist who needs to have specific expertise at a musical, relational, and clinical level. The interventions take place within a therapeutic setting and imply adequate methods of evaluation of the processes and of the results produced by them. The studies referring to $\mathrm{AMT}^{25,26,28,29,44-49}$ are mostly RCTs or CCTs, and as a consequence have a high level of evidence.

The relation-oriented approach of AMT can also be applied in interventions that involve the family caregivers (AMT-FC). In this case, the music therapist has a guiding role in facilitating the relationship between the PWD and important people in his/her familiar environment. The goal of this type of intervention is to enhance contact, communication, and connections, especially at a nonverbal level, between the PWD and the relative/caregiver. The intrinsic importance and value of this sound-music intervention are evident both on the level of emotion and affectivity and on the level of stress management by the relatives. The sound-music intervention represents a bridge that bypasses the cognitive obstacles and by doing so offers an alternative possibility to establish a relationship. The literature that is available in this field ${ }^{50-52}$ does not include RCTs or CCTs. This may be explained by the type of setting, which due to its characteristics and complexity cannot easily be represented by a scientific experimental model.

MBI indicates those activities that are not based on any specific music therapy model and that do not have specific therapeutic goals. Interventions of this kind are usually addressed to patient groups without the presence of a structured therapeutic setting. In this case, the presence of a qualified music therapist is not necessarily required, and the intervention usually consists of structured musical activities (eg, rhythmic use of instruments, singing, movements associated with music, listening to music). Studies describing this way of using music ${ }^{30,53-57}$ present methodological characteristics that are similar to the approaches based on music therapy (mostly RCTs or CCTs).

CS consists of singing and/or vocalism performed by the caregiver to promote contacts and relationships in the assistance of PWD. Moments of nursing and care can often cause stress, confusion, and disorientation to which the PWD responds with behavior that may be defensive, resisting, or aggressive in nature. Apart from the resulting uneasy situation, the caregiver also experiences difficulties and fatigue in exerting the nursing activities.

The singing activity can be carried out both by singing songs, ie, structured and organized sound-music material, and by vocalization (melodies without text), thus stimulating positive emotions in the interaction between caregiver and PWD. $42,58,59$

In the initial phase, this approach draws the PWD's attention toward the caregiver and gradually may favor the development of a positive relational climate that facilitates the different nursing activities (getting up in the morning, hygiene, getting dressed, escorting to the toilet, assistance in the bathing routine, handling of patients). ${ }^{60-63}$ This intervention can be carried out at the patient's home or in the nursing home. The person who performs the intervention can be both the formal (eg, nurse, operator) or the informal caregiver (relative, private assistant). The reported studies in this field do not include particularly big samples and are of a clearly qualitative nature.

The goal of ILM is to create personalized music listening that, on specific occasions, may gradually accompany the person toward a state of relaxation and greater well-being, 
thus reducing behavioral disturbances. Important descriptions of this type of intervention are given by the studies of Gerdner ${ }^{64,65}$ and Gerdner et $a 1^{66}$ but also by other researchers in this field. ${ }^{46,57,67-71}$ Information on the kind of music preferred by the patient is gained from relatives or from the contact person; ILM can be initiated by different formal or informal caregivers who have a relationship with the patient. The ILM intervention can be done at the patient's home as well as in the nursing home. The occasions on which ILM is preferably carried out are the moments of major discomfort and consequently when there are difficulties in adaptation to the environment, in communicating with other persons, and in performing the nursing activities. With regard to ILM, there are RCTs and CCTs, but the number of PWD involved in the studies are limited.

$\mathrm{BM}$ is defined by the use of prerecorded music in a certain environment in a precise situation (lunchtime, moments of rest) or in the absence of any specific activity. ${ }^{58,72-75}$ The goal of such music listening is the reduction of disturbed behavior and improvement of the general emotional atmosphere. BM is not directed to a single PWD but is proposed and diffused in the environment by the caregiver (following indications from the music therapist), generally in order to obtain activation or relaxation. Results emerging from the analysis of literature data on BM show little evidence, due to both the low number of persons involved and to the methods used in the studies.

\section{GMA-D}

The interventions described in our work introduce a possible GMA-D using music and sound-music elements according to the needs, clinical characteristics, and therapeutic-rehabilitation goals that emerge in the care of PWD. Table 1 reports and summarizes the main characteristics of interventions in the proposed model.

The GMA-D model offers the possibility to propose an approach that is strongly focused on the use of music at the moment of admission of the PWD.

This requires 1) an initial evaluation phase during which the music therapist and the physician focus their attention on the needs and the remaining capacities of the patient, 2) the definition of a music approach that is adapted to the PWD in accordance with practical evidence criteria, 3) a constant verification of the interventions and of their outcomes, and 4) the adjustment of the GMA-D in accordance with the clinical changes of the PWD and with the results that are obtained.

During the initial phase of dementia, for example, the patient can be more intensively stimulated by the different proposals provided by the model (AMT-PA, AMT-RA, MBI, ILM, and BM) according to the human resources and the needs of the PWD. When dementia worsens, the GMA-D model guides toward a reduction of the stimulation by introducing increasingly more individualized interventions, taking into consideration the aggravation of the physical, psychological, and cognitive conditions. In this respect, the interventions should be distributed in an equilibrated manner within the therapeutic-rehabilitation program in a way that the relational, communication, and behavioral problems observed, particularly during personal care procedures, are maximally supported (AMT-PA, AMT-FC, ILM, and CS). At the same time, interventions that are too arousing and/or require a level of cognitive and social-relational competence that is too high (AMT-FC, BM, and MBI) are gradually reduced.

Periodical evaluations are necessary in order to dispose of an updated clinical status and to direct and adjust the music program.

Furthermore, it is of fundamental importance to examine the response of the PWD to the single activities (by an evaluation grid to be filled in at each single session), but also to perform a periodical clinical assessment with the multidisciplinary team.

In view of this, the GMA-D model can be considered a flexible, dynamic, and adjustable approach that can be tailored to the PWD's needs.

Besides, the possibility to overlap several interventions with music allows adjusting the stimulation in an adequate manner, hereby potentially reinforcing the awaited effects.

\section{The feasibility and the implementation of the model in real-world settings}

The practical implementation of the GMA-D model requires the presence of a music therapist (adequately trained and with a profound knowledge of the clinical cases to be treated) who, in collaboration with the involved clinical staff, guarantees the correct planning and management of the interventions and their evaluation. Therefore, the music therapist will be directly involved in some of the interventions (AMT-PA, AMT-RA, and AMT-FC), while in others (MBI, CS, ILM, and $\mathrm{BM}$ ) he/she will prepare the intervention project and will be responsible for the specific training and supervision of the health workers and caregivers involved. As a consequence, the feasibility of the GMA-D model depends on the establishment of a working group capable of sharing knowledge, skills, and goals. 


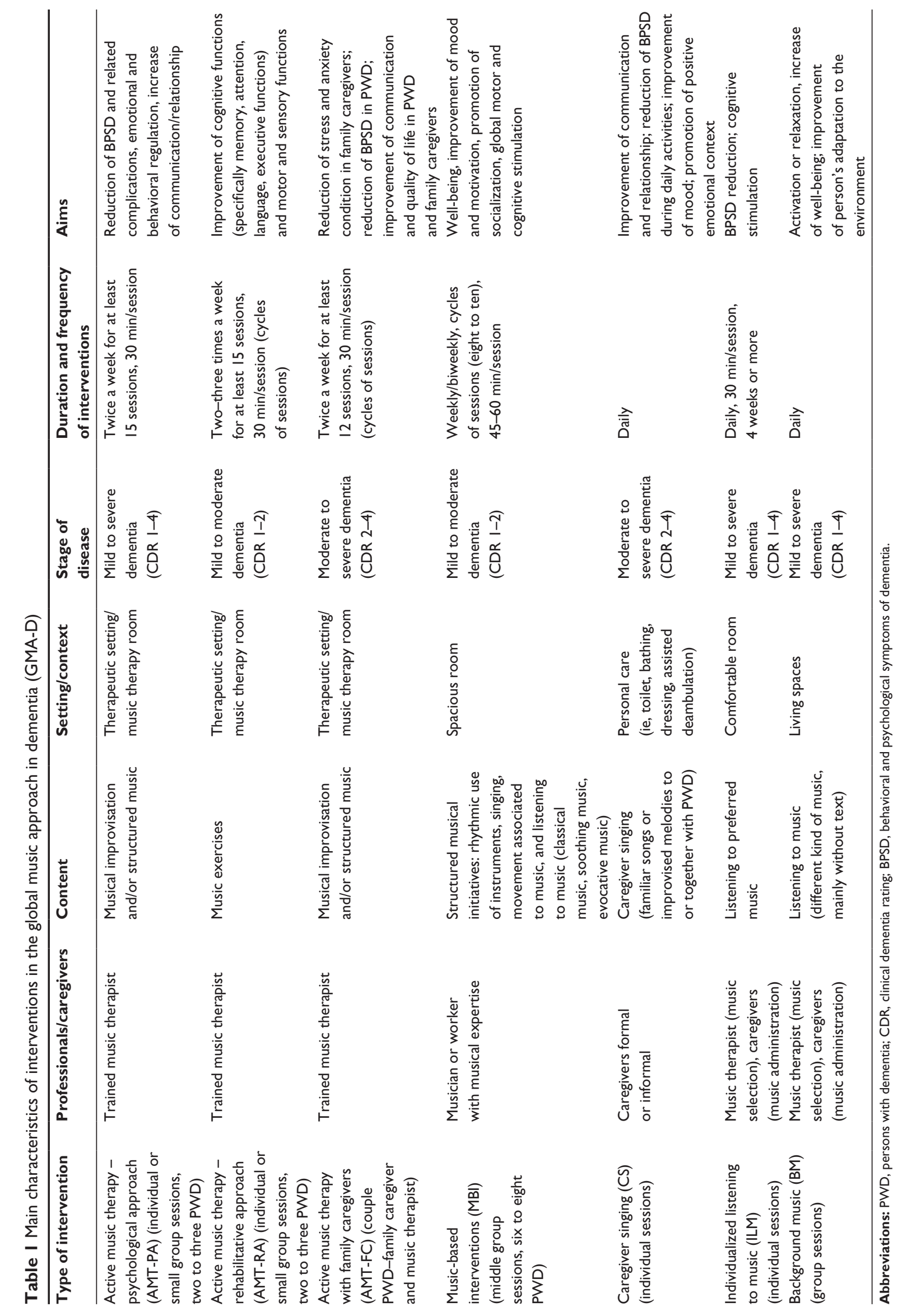


Standardizing the operational methods of the single activities and the instruments for the evaluation of the interventions and their results will be of fundamental importance. From this point of view, the identification of a joint practice can contribute to achieving specific evaluation methods that can be validated within the application model.

Also, from a clinical viewpoint, it is possible to identify specific clinical instruments to examine the results of GMA-D.

Besides the clinical implementation of GMA-D, it will also be important to obtain further evidence for those interventions (especially AMT-RA, AMT-FC, CS, and BM) that have not yet been examined by scientifically structured studies (RCTs or CCTs).

The evidence-based approach used for implementing the model may be a valid starting point for sharing content, operational methods, and research perspectives.

It will be extremely important to create a network that combines the international experiences obtained with this model by the creation of a database in which intervention protocols and the results of their application can be archived and made available for consulting.

The ongoing experience with the single interventions and with the use of the GMA-D model in Italy shows interesting confirmations from a clinical point of view regarding the management of dementia-related issues.

The GMA-D model is also to be considered a low-cost nonpharmacological intervention and a therapeutic-rehabilitation method for the reduction of behavioral and psychological symptoms of dementia, for stimulation of cognitive functions, and for increasing the overall quality of life of the PWD.

\section{Acknowledgments}

Special thanks to the Director Dr Mariarosa Dossi, the President Renzo Dori, and to the staff of the Azienda Pubblica di Servizi alla Persona "Margherita Grazioli" of Povo (TN, Italy) for their contribution and support in the design and construction of the GMA-D model.

\section{Author contributions}

AR developed the study design concept and wrote the manuscript; SF developed the study design concept and contributed to searching the literature and to the writing; MSB and DB contributed to analyzing and interpreting the data, and to writing the manuscript and revised it. All authors read and approved the final version of the manuscript.

\section{Disclosure}

The authors report no conflicts of interest in this work.

\section{References}

1. Koger SM, Brotons M. Music therapy for dementia symptoms. Cochrane Database Syst Rev. 2000;(3):CD001121.

2. Sherratt K, Thornton A, Hatton C. Music interventions for people with dementia: a review of the literature. Aging Ment Health. 2004;8(1): $3-12$.

3. Vink AC, Birks JS, Bruinsma MS, Scholten RJ. Music therapy for people with dementia. Cochrane Database Syst Rev. 2004;(3):CD003477.

4. Raglio A, Bellelli G, Mazzola P, et al. Music, music therapy and dementia: a review of literature and the recommendations of the Italian psychogeriatric association. Maturitas. 2012;72:305-310.

5. McDermott O, Crellin N, Ridder HM, Orrell M. Music therapy in dementia: a narrative synthesis systematic review. Int $J$ Geriatr Psychiatry. 2013;28(8):781-794.

6. Raglio A, Gianelli MV. Music and music therapy in the management of behavioral disorders in dementia. Neurodegener Dis Manag. 2013;3(4):295-298.

7. Robb SL, Burns DS, Carpenter JS. Reporting guidelines for music-based interventions. Music Med. 2011;3(4):271-279.

8. Vink A, Bruinsma M. Evidence Based Music Therapy. Available from: http://www.wfmt.info/Musictherapyworld/modules/mmmagazine/issu es/20031103132043/20031103134548/Vink.pdf. Accessed September 8, 2014.

9. Koelsch S. A neuroscientific perspective on music therapy. Ann $N Y$ Acad Sci. 2009;1169:374-384.

10. Koelsch S. Towards a neural basis of music-evoked emotions. Trends Cogn Sci. 2010;14(3):131-137.

11. Levitin DJ, Tirovolas AK. Current advances in the cognitive neuroscience of music. Ann N Y Acad Sci. 2009;1156:211-231.

12. Zatorre R. Music and the brain. Ann N Y Acad Sci. 2003;999:4-14.

13. Zatorre R, McGill J. Music, the food of neuroscience? Nature. 2005; 17(7031):312-315.

14. Koelsch S. Brain correlates of music-evoked emotions. Nat Rev Neurosci. 2014;15(3):170-180.

15. Schlaug G. Part VI introduction: listening to and making music facilitates brain recovery processes. Ann N Y Acad Sci. 2009;1169:372-373.

16. Sloboda J. Exploring the Musical Mind: Cognition, Emotion, Ability, Function. Oxford, UK: Oxford University Press; 2005.

17. Chanda ML, Levitin DJ. The neurochemistry of music. Trends Cogn Sci. 2013;17(4):179-193.

18. Loomba RS, Arora R, Shah PH, Chandrasekar S, Molnar J. Effects of music on systolic blood pressure, diastolic blood pressure, and heart rate: a meta-analysis. Indian Heart J. 2012;64(3):309-313.

19. Okada K, Kurita A, Takase B, et al. Effects of music therapy on autonomic nervous system activity, incidence of heart failure events, and plasma cytokine and catecholamine levels in elderly patients with cerebrovascular disease and dementia. Int Heart J. 2009;50(1):95-110.

20. Raglio A, Oasi O, Gianotti M, et al. Effects of music therapy on psychological symptoms and hearth rate variability in patients with dementia. A pilot study. Curr Aging Sci. 2010;3(3):242-246.

21. Takahashi T, Matsushita H. Long-term effect of music therapy on elderly with moderate-severe dementia. J Music Ther. 2006;43(4):317-333.

22. Gold C, Solli HP, Kruger V, Lie SA. Dose-response relationship in music therapy for people with serious mental disorders; systematic review and meta-analysis. Clin Psychol Rev. 2009;29(3): 193-207.

23. Trevarthen C, Aitken KJ. Infant intersubjectivity: research, theory and clinical application. J Child Psychol Psychiatry. 2001;42(1):3-48.

24. Wigram T. Improvisation: Methods and Techniques for Music Therapy Clinicians, Educators and Students. London, UK: Jessica Kingsley Publishers; 2004.

25. Raglio A, Bellelli G, Traficante D, et al. Efficacy of music therapy treatment based on cycles of sessions: a randomized controlled trial. Aging Ment Health. 2010;14(8):900-904.

26. Raglio A, Bellelli G, Traficante D, et al. Efficacy of music therapy in the treatment of behavioral and psychiatric symptoms of dementia. Alzheimer Dis Assoc Disord. 2008;22(2):158-162. 
27. Ueda T, Suzukamo Y, Sato M, Izumi SI. Effects of music therapy on behavioral and psychological symptoms of dementia: a systematic review and meta-analysis. Ageing Res Rev. 2013;12(2):628-641.

28. Brotons M, Koger SM. The impact of music therapy on language functioning in dementia. J Music Ther. 2000;37(3):183-195.

29. Ceccato E, Vigato G, Bonetto C, et al. STAM protocol in dementia: a multicenter, single-blind, randomized, and controlled trial. Am J Alzheimers Dis Other Demen. 2012;27(5):301-310.

30. Van de Winckel A, Feys H, De Weerdt W, Dom R. Cognitive and behavioral effects of music-based exercises in patients with dementia. Clin Rehabil. 2004;18(3):253-260.

31. Cowles A, Beatty WW, Nixon SJ, Lutz LJ, Paulk J, Paulk K. Musical skill in dementia: a violinist presumed to have Alzheimer's disease learns to play a new song. Neurocase. 2003;9:493-503.

32. Cuddy LL, Duffin J. Music memory and Alzheimer's disease: is music recognition spared in dementia, and how can it be assessed? Med Hypotheses. 2005;64(2):229-235.

33. Fornazzari L, Castle T, Nadkarni S, et al. Preservation of episodic musical memory in a pianist with Alzheimer disease. Neurology. 2006;66(4):610-611.

34. Raglio A, Gianelli MV. Music therapy for individuals with dementia: areas of intervention and research perspective. Curr Alzheimer Res. 2009;6(3):293-301.

35. Vasionytė I, Madison G. Musical intervention for patients with dementia: a meta-analysis. J Clin Nurs. 2013;22(9-10):1203-1216.

36. Wall M, Duffy A. The effects of music therapy for older people with dementia. Br J Nurs. 2010;19(2):108-113.

37. Skingley A, Vella-Burrows T. Therapeutic effects of music and singing for older people. Nurs Stand. 2010;24(19):35-41.

38. Sorrell JA, Sorrell JM. Music as a healing art for older adults. J Psychosoc Nurs Ment Health Serv. 2008;46(3):21-24.

39. Goodall D, Etters L. The therapeutic use of music on agitated behavior in those with dementia. Holist Nurs Pract. 2005;19(6):258-262.

40. Lou MF. The use of music to decrease agitated behaviour of the demented elderly: the state of the science. Scand J Caring Sci. 2001;15(2):165-173.

41. Guetin S, Charras K, Berard A, et al. An overview of the use of music therapy in the context of Alzheimer's disease: a report of a French expert group. Dementia (London). 2013;12(5):619-634.

42. Hammar LM, Emami A, Engström G, Götell E. Communicating through caregiver singing during morning care situations in dementia care. Scand J Caring Sci. 2011;25(1):160-168.

43. Sung HC, Chang AM. Use of preferred music to decrease agitated behaviours in older people with dementia: a review of the literature. $J$ Clin Nurs. 2005;14(9):1133-1140.

44. Choi AN, Lee MS, Cheong KJ, Lee JS. Effects of group music intervention on behavioral and psychological symptoms in patients with dementia: a pilot-controlled trial. Int J Neurosci. 2009;119(4):471-481.

45. Chu H, Yang CY, Lin Y, et al. The impact of group music therapy on depression and cognition in elderly persons with dementia: a randomized controlled study. Biol Res Nurs. 2014;16(2):209-217.

46. Raglio A, Bellandi D, Baiardi P, Gianotti M, Ubezio MC, Granieri E. Listening to music and active music therapy in behavioral disturbances in dementia: a crossover study. J Am Geriatr Soc. 2013;61(4):645-647.

47. Ridder HMO, Stige B, Gunnhild L. Individual music therapy for agitation in dementia: an exploratory randomized controlled trial. Aging Ment Health. 2013;17(6):667-678.

48. Svansdottir HB, Snaedal J. Music therapy in moderate and severe dementia of Alzheimer's type: a case-control study. Int Psychogeriatr. 2006;18(4):613-621.

49. Vink AC, Zuidersma M, Boersma F, de Jonge P, Zuidema SU, Slaets JP. The effect of music therapy compared with general recreational activities in reducing agitation in people with dementia: a randomised controlled trial. Int J Geriatr Psychiatry. 2013;28(10): 1031-1038.

50. Brotons M, Marti P. Music therapy with Alzheimer's patients and their family caregivers: a pilot project. J Music Ther. 2003;40(2):138-150.
51. Clair A. The effects of music therapy on engagement in family caregiver and care receiver couples with dementia. Am J Alzheimers Dis Other Demen. 2002;17(5):286-290.

52. Hanser SB, Butterfield-Whitcomb J, Kawata M, Collins BE. Homebased music strategies with individuals who have dementia and their family caregivers. J Music Ther. 2011;48(1):2-27.

53. Sung HC, Chang SM, Lee MS. The effect of group music with movement intervention on agitated behaviors of institutionalized elders with dementia in Taiwan. Complement Ther Med. 2006;14(2):113-119.

54. Cooke Ml, Moyle W, Shum DH, Harrison SD, Murfield J. A randomized controlled trial exploring the effect of music on quality of life and depression in older people with dementia. J Health Psychol. 2010;15(5):765-776.

55. Lin Y, Chu H, Yang CY, et al. Effectiveness of group music intervention against agitated behavior in elderly persons with dementia. Int $J$ Geriatr Psychiatry. 2011;26(7):670-678.

56. Sung H, Lee W, Li T, Watson R. A group music intervention using percussion instruments with familiar music to reduce anxiety and agitation of institutionalized older adults with dementia. Int $J$ Geriatr Psychiatry. 2012;27(6):621-627.

57. Sakamoto M, Ando H, Tsutou A. Comparing the effects of different Individualized music interventions for elderly individuals with severe dementia. Int Psychogeriatr. 2013;25(5):775-784.

58. Götell E, Brown S, Ekman SL. Caregiver singing and background music in dementia care. West J Nurs Res. 2002;24(2):195-216.

59. Chatterton W, Baker F, Morgan $\mathrm{K}$. The singer or the singing: who sings individually to persons with dementia and what are the effects? Am J Alzheimers Dis Other Demen. 2010;25(8):641-649.

60. Götell E, Brown S, Ekman SL. Influence of caregiver singing and background music on posture, movement and sensory awareness in dementia care. Int Psychogeriatr. 2003;15(4):411-430.

61. Götell E, Brown S, Ekman SL. The influence of caregiver singing and background music on vocally expressed emotions and moods in dementia care: a qualitative analysis. Int J Nurs Stud. 2009;46(4):422-430.

62. Hammar LM, Emami A, Engström G, Götell E. Reactions of persons with dementia to caregivers singing in morning care situations. Open Nurs J. 2010;4:35-41

63. Hammar LM, Emami A, Götell E, Engström G. The impact of caregivers' singing on expressions of emotion and resistance during morning care situations in persons with dementia: an intervention in dementia care. J Clin Nurs. 2011;20(7-8):969-978.

64. Gerdner LA. An individualized music intervention for agitation. J Am Psychiatr Nurses Assoc. 1997;3:177-184.

65. Gerdner LA. Effects of individualized versus classical "relaxation" music on the frequency of agitation in elderly persons with Alzheimer's disease and related disorders. Int Psychogeriatr. 2000;12(1):49-65.

66. Gerdner LA, Hamed SA, Elserogy YB, Abdou MA, Abdellah MM. Individualized music for dementia: evolution and application of evidence-based protocol. World J Psychiatry. 2012;2(2):26-32.

67. Clark ME, Lipe AW, Bilbrey M. Use of music to decrease aggressive behaviors in people with dementia. J Gerontol Nurs. 1998;24(7):10-17.

68. Ragneskog H, Asplund K, Kihlgren M, Norberg A. Individualized music played for agitated patients with dementia: analysis of video-recorded sessions. Int J Nurs Pract. 2001;7(3):146-155.

69. Sung HC, Chang AM, Abbey J. The effect of preferred music on agitation of older people with dementia in Taiwan. Int J Geriatr Psychiatry. 2006;21(10):999-1000.

70. Park H, Pringle Specht JK. Effect of individualized music on agitation in individuals with dementia who live at home. J Gerontol Nurs. 2009;35(8):47-55.

71. Sung HC, Chang SM, Lee MS. A preferred music listening intervention to reduce anxiety in older adults with dementia in nursing homes. Clin Nurs. 2010;19(7-8):1056-1064.

72. Ragneskog H, Brane G, Karlsson I, Kihlgren M. Influence of dinner music on food intake and symptoms common in dementia. Scand $J$ Caring Sci. 1996;10(1):11-17. 
73. Ziv N, Granot A, Ha IS, Dassa A, Haimov I. The effect of background stimulative music on behavior in Alzheimer's patients. J Music Ther. 2007;44(4):329-343.

74. Chang FY, Huang HC, Lin KC, Lin LC. The effect of a music programme during lunchtime on the problem behaviour of the older residents with dementia at an institution in Taiwan. J Clin Nurs. 2010;19(7-8):939-948.
75. Dunn KS, Riley-Doucet CK. Comparative analysis of two musical genres within a multisensory environmental intervention. J Holist Nurs. 2013;31(1):62-70.

\section{Publish your work in this journal}

Clinical Interventions in Aging is an international, peer-reviewed journal focusing on evidence-based reports on the value or lack thereof of treatments intended to prevent or delay the onset of maladaptive correlates of aging in human beings. This journal is indexed on PubMed Central, MedLine,
CAS, Scopus and the Elsevier Bibliographic databases. The manuscript management system is completely online and includes a very quick and fair peer-review system, which is all easy to use. Visit http://www.dovepress. $\mathrm{com} /$ testimonials.php to read real quotes from published authors. 